\title{
Development of a test system for evaluating the antiglycative effect of bioactive substances of medicinal plants
}

\author{
Vashurina N.V. ${ }^{1 *}$, Dubrovskii Y.A. ${ }^{2,3}$, Soboleva A.V. ${ }^{1,4}$, Frolov A.A. ${ }^{1,4}$ \\ ${ }^{I}$ Department of Biochemistry, Saint Petersburg State University, Saint Petersburg, Russia \\ ${ }^{2}$ Department of Chemistry, Saint Petersburg State University, Saint Petersburg, Russia \\ ${ }^{3}$ Almazov National Medical Research Centre, Saint Petersburg, Russia \\ ${ }^{4}$ Department of Bioorganic Chemistry, Leibniz Institute of Plant Biochemistry, Halle (Saale), Germany
}

Key words: protein glycation, medicinal plants, incubations

Motivation and Aim: Protein glycation and formation of advanced glycation end products (AGEs) essentially impact on complications of diabetes mellitus, pathogenesis of Alzheimer's disease and cancer. In this context, chemical substances, inhibiting protein glycation might be promising, anti-diabetic and neuroprotective agents. Indeed, to date several anti-glycative drugs, such as aminoguanidine, have been shown to be effective in therapy of diabetes complications. However, these drugs are highly toxic, that limits their clinical application. In this context, plant natural products, isolated from medicinal plants offer a rich source of new promissing candidates for pharma- and nutraceutical agents, able to inhibit AGE formation. However, for fast and efficient screening of extracts, fractions and individual compounds, and adequate reproducible and high-through test system is necessary.

Methods and Algorithms: The proposed test system will rely on in vitro incubations $(1,3,7,14$ and 21 days) of plant extracts and individual natural products with Maillard reaction mixtures. These mixtures will contain $0.5 \mathrm{mmol} / \mathrm{L} D$-glucose in sodium phosphate buffer $(\mathrm{pH} 7.2)$ and equimolar amounts of model synthetic peptides Ac-AFGSAKASGA-NH $\mathrm{N}_{2}$ and Ac-AFGSARASGA-NH $\mathrm{N}_{2}$ used in the previous work [1]. The progress of glycation can be followed by accumulation of individual AGEs at defined glycation sites, using liquid chromatography, coupled on-line to mass spectrometry (LC-MS). The applicability of the test system for determination of anti-glycative effects will be verified with aqueous and ethyl acetate extracts $(n=4)$ of the aerial parts of Geum rivale taken at a non-cytotoxic concentration of $33.3 \mu \mathrm{g} / \mathrm{ml}$. Thereby, the compounds with known anti-glycative effect rosmarinic acid at the concentration of $0.4 \mathrm{mg} / \mathrm{mL}$ and aminoguandine at the concentration of $5 \mathrm{mmol} / \mathrm{L}$ will be used as reference compounds.

Conclusion and outlook: At the next step, a representative set of medicinal plants will be tested. For this, 22 Astragalus species, collected from different regions of Uzbekistan will be considered. Methanolic extracts will be prepared and analyzed by UHPLC-QqTOF-MS in parallel to screening for anti-glycative effects. Prior to the probing anti-AGE properties, maximal non-toxic extract concentration will be determined in cell viability tests (3-(4,5-dimethylthiazol-2-yl)-2,5-diphenyltetrazolium bromide (MTT) assay) with SH-SY5Y human neuroblastoma cells using established methods [2].

\section{References}

1. Bilova T. et al. J. Biol. Chem. 2016. 291. 7621-7636.

2. Leonova T. et al. Int. J. Mol. Sci. 2020. 21. 